\title{
Post PCNL Renal Pseudoaneurysm and AV Fistula: Single Institution Analysis
}

\section{Ajay Anand ${ }^{*}$ and Amit Manhas}

Department of Urology and Surgery, Superspeciality Hospital, Govt. Medical College Hospital, Jammu, India

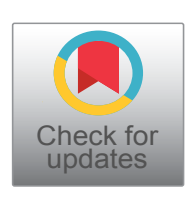

*Corresponding author: Dr. Ajay Anand, Associate Professor, Department of Urology and Surgery, Super Speciality Hospital, Government Medical College Hospital, Jammu-180001, India, Tel: 91-191-2584290

\begin{abstract}
Objectives: To assess post-PCNL causes of hematuria and their best treatment.

Subjects and Methods: A total of 112 patients underwent PCNL between February 2016 till date through tract sizes ranging from $22 \mathrm{Fr}$ to $26 \mathrm{Fr}$.

Results: Seven patients out of a total of 24 patients with superior calyceal puncture developed post-PCNL hematuria. Only one patient could be managed conservatively while rest 6 required angiography and angioembolization to control hematuria.

Conclusions: Early diagnosis and treatment of post-PCNL hematuria is warranted for a successful postoperative outcome with minimal morbidity.
\end{abstract}

\section{Keywords}

PCNL, Hematuria, Angioembolization

\section{Abbreviations}

PCNL: Percutaneous Nephrolithotomy; Fr: French; A-V Fistula: Arteriovenous Fistula; APF: Arteriopelvic Fistula

\section{Introduction}

PCNL has been accepted as a minimally invasive gold standard treatment for renal calculi. Though PCNL is a minimally morbid procedure yet it is fraught with rare but significant complication of post-procedural hematuria. Massive hematuria due to renovascular injury has been reported in $0.3-1 \%$ of patients following PCNL [1]. Persistent hematuria can be life-threatening if not managed on emergency basis. Almost all renal punctures for PCNL are associated with vascular injury - some trivial and self-resolving; while other injuries can be progressive leading to pseudoaneurysms or A-V fistulas, which need to be investigated and managed by angioembolization.

Type of renovascular injury can be detected either by angiography [2] or multidimensional computerized tomography angiogram with 3-D reconstruction.

\section{Subjects and Methods}

We prospectively followed patients of PCNL in Government Medical College Hospital, Jammu starting from February 2016 till date. All patients of renal calculi ranging from simple renal calculus to complete staghorn calculi were treated with PCNL at our institution.

All patients underwent PCNL under general anaesthesia, during period February 2016 till date. Standard protocol as used for PCNL was followed. Preferential renal puncture for PCNL was guided by stone location and size. Tract dilatation was performed using metal dilators in all the cases. Tract size ranged from $22 \mathrm{Fr}$ to $26 \mathrm{Fr}$ depending on hydronephrosis and stone bulk. Pneumatic lithotripter was used for in situ lithotripsy. Initial calyceal puncture was performed with C-arm at 30 degree towards the surgeon; and depth of needle was confirmed with $\mathrm{C}$-arm at 0 degree. In some cases with migrated stones, bull's eye puncture was used to puncture wash and retrieve the fragments through main puncture. Double J stent was kept in all patients. Nephrostomy tube was placed in all cases postoperatively. Stone clearance was confirmed on C-arm intraoperatively and X-ray KUB on first postoperative day. Nephrostomy tube was removed on $2^{\text {nd }}$ to $3^{\text {rd }}$ postoperative day when urine was clear. Indwelling catheter was removed on succeeding day after nephrostomy remov- 
al. Double J stent was removed after three weeks postoperatively. We kept double J stent in all of our patients under study. It ensured dual purpose of adequate distal

Table 1: Patient demographics.

\begin{tabular}{|l|l|}
\hline Total number of patients & 112 \\
\hline Age (years) & $43.27(15-81)$ \\
\hline Male : Female & $57: 55$ \\
\hline Average stone diameter $(\mathrm{mm})$, mean \pm SD & $31.23 \pm 19.10$ \\
\hline $\begin{array}{l}\text { Number of patients with simple renal pelvic } \\
\text { or calyceal calculus }\end{array}$ & $59(52.7 \%)$ \\
\hline Number of patients with staghorn calculi & $53(47.3 \%)$ \\
\hline
\end{tabular}

Table 2: Intraoperative parameters.

\begin{tabular}{|l|l|}
\hline Parameter & Number of patients (\%) \\
\hline Tract size 22 Fr & $27(24.1 \%)$ \\
\hline Tract size 24 Fr & $85(75.9 \%)$ \\
\hline $\begin{array}{l}\text { Number of patients with } \\
\text { superior calyceal puncture }\end{array}$ & $24(21.4 \%)$ \\
\hline $\begin{array}{l}\text { Post-PCNL hematuria in } \\
\text { superior calyceal punctures }\end{array}$ & $07 / 24(29.2 \%)$ \\
\hline
\end{tabular}

Table 3: Postoperative parameters.

\begin{tabular}{|l|l|}
\hline Parameter & Number of patients (\%) \\
\hline Significant hematuria & $7(29.2 \%)$ \\
\hline $\begin{array}{l}\text { Angiography and selective } \\
\text { angioembolization }\end{array}$ & $6(25 \%)$ \\
\hline A-V fistula & $2(8.3 \%)$ \\
\hline Conservative & $1(4.2 \%)$ \\
\hline Postoperative Follow-up & Uneventful \\
\hline
\end{tabular}

drainage postoperatively and decreased incidence of urine leak after nephrostomy tube removal.

In patients with persistent hematuria, nephrostomy tube was clamped immediately to allow clot formation and tamponade for a day or two, till urine got clear. Persistent hematuria requiring two or more units of blood transfusion were investigated using angiography and cases with pseudoaneurysms or A-V fistula were managed by selective or superselective embolization using micro-coils.

\section{Results}

The patient characteristics and intraoperative parameters are shown in Table 1 and Table 2. A total of 112 patients underwent PCNL. Age of patients ranged from 15-81 years with mean age being 43.27 years. There were 57 males and 55 females. Average stone diameter was $31.23 \pm 19.10 \mathrm{~mm}$. 7 patients had previous history of open surgery and 1 patient had prior history of PCNL on same side.

There were 59 patients with simple renal or pelvic calculus and 53 patients had staghorn calculi. Out of staghorn calculi, 9 patients had complete staghorn calculi which needed relook procedure through same as well as additional tracts. Tract size was $22 \mathrm{Fr}$ in 27 patients. 24 patients underwent PCNL through superior calyceal puncture. Tract size in patients with superior calyceal puncture was $24 \mathrm{Fr}$ in all cases.

Postoperative parameters of patients are shown in Table 3. Seven out of these 24 patients $(29.2 \%)$ with su-

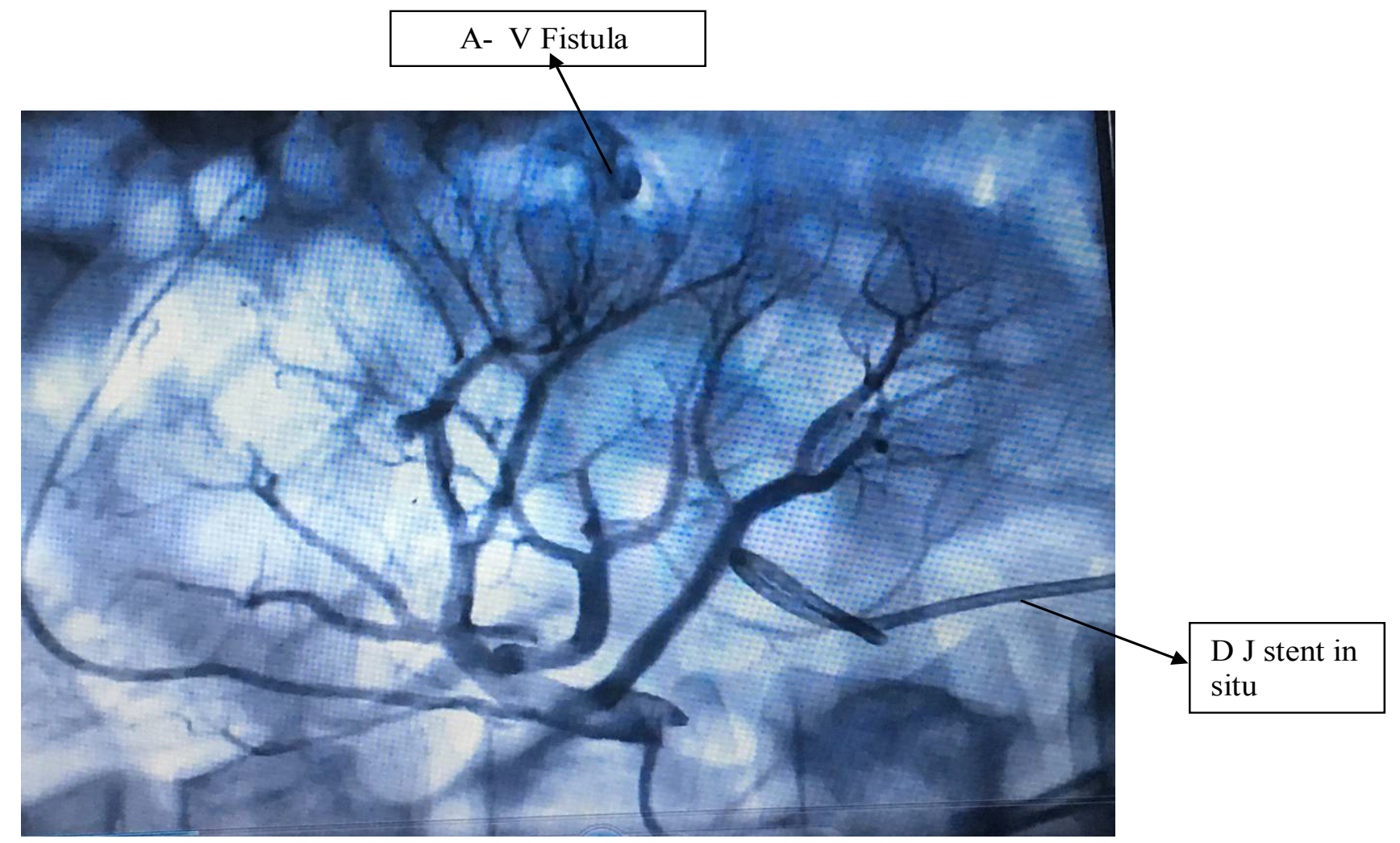

Figure 1: Angiogram showing A-V Fistula post-PCNL treated by selective embolization using micro-coils. 
perior calyceal puncture had significant postoperative hematuria, necessitating intervention. Six out of these seven patients $(25 \%)$ underwent angiography and selective embolization. Two of these patients (8.3\%) had A-V fistula on angiography as shown in Figure 1. One patient $(4.2 \%)$ was managed conservatively and his hematuria settled with uneventful outcome on subsequent follow-up. Clavien-Dindo classification system, devised in 2004 was used to grade these injuries. One patient who was managed conservatively with blood transfusion had grade II injury while six patients who had to undergo angiography and embolization were grade IIla injuries as per this classification.

\section{Discussion}

PCNL is basically a grade 3 renal trauma and trivial to significant vascular injury is encountered in almost all patients. Some trivial injuries do not need any intervention and resolve on their own. However some renovascular injuries are significant and need further investigation and treatment so as to control the haemorrhage. We define post-PCNL hematuria as significant if patient requires two or more units of blood transfusion postoperatively.

These renovascular injuries can be immediate, early or delayed. Immediate injuries are usually managed by clamping nephrostomy tubes postoperatively so as to allow tamponade of bleeder and clot formation. Trivial injuries settle with this conservative trial in a day or two. Early injuries are encountered after two to three days and may be because of pseudoaneurysm formation or A-V fistula. Delayed injuries are rare to encounter. They are usually because of pseudoaneurysm rupture or infection.

The reported incidence of renal pseudoaneurysm following PCNL is $0.6-1 \%$ [3]. In less severe cases, bleeding arises from the venous channels or an infection of necrosed parenchymal tissue. Venous bleeding can usually be managed conservatively because the intrarenal venous system is quite resilient; whereas, arterial bleeding needs to be embolized.

Such post-PCNL renovascular injuries need urgent intervention. This can be in form of angiography and angioembolization as was done in 6 of our cases under study. Selective angioembolization was performed in our cases with vascular injury. Angioembolization can be either superselective or selective. Other non-invasive modality which can be used to diagnose vascular injury can be multidimensional computerized tomogram angiogram with 3-D reconstruction [4].

Owing to good role of MDCT in identifying the source of acute gastrointestinal hemorrhage, there is an enthusiasm for its applications in other specialities [5]. Besides being non invasive and having short scanning times, MDCT provides thinner collimation, greater anatomic coverage, and better multi-planar reformatted images expanding its diagnostic role for various pathologic processes. As opposed to angiography, MDCT offers information not only regarding the site of bleeding but also of the details regarding perinephric hematoma, residual stones, urinary extravasation, and hydronephrosis. It also allows improved interventional planning and lesion directed treatment. Since the diagnosis is being established prior to intervention, the total interventional time is reduced thereby decreasing the radiation exposure to the patient and the radiologist.

By convention, a selective angiogram has been used as a primary investigation when life-threatening bleeding occurs after PCNL. Although this gives the advantage of being able to perform an immediate therapeutic embolization, there may be limitations with respect to an accurate diagnosis. First, it may reveal the cause of hematuria only when there is active bleeding; intermittent or delayed bleeding, which usually occurs in pseudoaneurysms, may be missed during the interval time. Secondly, venous bleeding and bleeding associated with infection may not be readily detected by conventional angiograms. Finally, being invasive in nature, there is an overall complication rate of $3.8 \%$ (1.3\% major) due to vascular access for all diagnostic arteriographic procedures [6].

There has been no significant difference in overall complications and blood transfusion rates, via middle calyceal access or lower calyceal access for isolated lower pole kidney stones [7]. Although superior calyceal access breaches diaphragm and is believed to have greater complications than access through other calyces; yet superior calyceal puncture provides direct access to pelvis and all calyces, with minimal torquing and better stone clearance rates [8]. It has been observed that if puncture is made through upper pole infundibulum, then in $67 \%$ of the kidneys interlobar vessels are injured while if lower pole infundibular puncture was made then only in $13 \%$ of the kidneys interlobar vessels are injured $[9,10]$. We preferred stone guided puncture; with preferential superior calyceal access for staghorn calculi.

There is a very rare cause of post-PCNL hematuria because of arteriopelvic fistula (APF). This has not been extensively studied but has been reported to be managed using ureteroscopic localization and fibrin sealant placement at fistula site [11].

There have been reports of post PCNL bleeding attributable to AV fistulas and pseudoaneurysms. Massive bleeding can occur in the early postoperative period ( 2 to 14 days after PCNL), and arteriovenous fistula formation as a late complication 40 days after PCNL [12]. We also observed bleeding because of AV fistula in 2 (8.3\%) patients of superior calyceal access. Both these patients presented 5-10 days after PCNL. AV fistulas were diagnosed on angiography and successfully managed using microcoils. 
Studies have reported failure of initial superselective and selective renal artery embolization for post-PCNL haemorrhage. 3 risk factors have been identified for failure of initial superselective renal arterial embolization, including multiple percutaneous access sites, more than 2 bleeding sites identified on renal angiogram and gelatin sponge alone used as the embolic material. Authors opined carefully selecting patients for multitract percutaneous nephrolithotomy, making an extra effort to identify all bleeding vessels during angiography and not using gelatin sponge as the only embolic material could potentially decrease the risk of failure of initial superselective renal arterial embolization after percutaneous nephrolithotomy [13]. Another study identified large tract size, multiple bleeding sites, renal vascular aberration/tortuosity as significant risk factors for increased risk of initial treatment failure of superselective renal arterial embolization [14]. All our patients were successfully managed by initial embolization. We recommend use of microcoils as an embolizing agent.

There are standard protocols to manage post PCNL haemorrhage. Monitor vital signs and obtain good venous access with blood transfusion. Colloids can be administered till blood is arranged. If nephrostomy tube is present, it is clamped for tamponade effect. If gross hematuria with acute urine retention occurs, Foley catheterization with urinary bladder irrigation should be administered. Hydration and intravenous administration of mannitol in hemodynamically stable patients can lead to rapid forced diuresis and swelling of the kidney within the capsule, which may enhance tract tamponade. Persistence of haemorrhage and need for more than two blood transfusions warrants early angiography and embolization; preferably using microcoils.

\section{Conclusion}

Early diagnosis and treatment of post-PCNL hematuria is warranted to reduce morbidity and a successful postoperative outcome.

\section{Compliance with Ethical Requirements}

This manuscript meets ethical standard requirements.

\section{Acknowledgement}

I, Ajay Anand declare that there is no conflict of interest. There are no financial implications involved and no grants taken from any organization in research and drafting of this manuscript.

\section{References}

1. Martin X, Murat FJ, Feitosa LC, Rouviére $O$, Lyonnet $D$, et al. (2000) Severe bleeding after nephrolithotomy: Results of hyper selective embolization. Eur Urol 37: 136-139.

2. El-Nahas AR, Shokeir AA, El-Assmy AM, Mohsen T, Shoma AM, et al. (2007) Post-percutaneous nephrolithotomy extensive hemorrhage: A study of risk factors. J Urol 177: 576-579.
3. Massulo-Aguiar MF, Campos CM, Rodrigues-Netto N Jr (2006) Intrarenal pseudoaneurysm after percutaneous nephrolithotomy angiotomographic assessment and endovascular management. Int Braz J Urol 32: 440-444.

4. SE Sivanandam, Georgie Mathew, Sanjay H Bhat (2009) Emerging role of multi-detector computed tomography in the diagnosis of hematuria following percutaneous nephrolithotomy: A case scenario. Indian J Urol 25: 392-394.

5. Laing CJ, Tobias T, Rosenblum DI, Banker WL, Tseng L, et al. (2007) Acute gastrointestinal bleeding: Emerging role of multidetector CT angiography and review of current imaging techniques. Radiographics 27: 1055-1070.

6. AbuRahma AF, Elmore M, Deel J, Mullins B, Hayes J (2007) Complications of diagnostic arteriography performed by a vascular surgeon in a recent series of 558 patients. Vascular 15: 92-97.

7. Caglayan V, Onen E, Avci S, Kilic M, Sambel M, et al. (2020) Percutaneous nephrolithotomy via a middle calyx access is effective in the treatment of lower pole kidney stones: A single-center study. Urol Int 21: 1-5.

8. Ajay Anand, Rajeev Kumar, Prem N Dogra, Amlesh Seth, Narmada P Gupta (2010) Safety and efficacy of a superior caliceal puncture in pediatric percutaneous nephrolithotomy. J Endourol 24: 1725-1728.

9. Sampaio FJB, Zanier JFC, Aragao AHM, Favorito LA (1992) Intrarenal access: Three- dimensional anatomic study. J Urol 148: 1769-1773.

10. Mufti Mahmood Ahmed, Fayaz Ahmad Najar (2019) Safety and efficacy of supracostal superior calyceal approach for percutaneous renal surgery. Turk J Urol 45: S121-S124.

11. Danny Chao, Alym N Abdulla, Soojin Kim, Jen Hoogenes, Edward D Matsumoto (2014) A novel endoscopic treatment for renal arteriopelvic fistula post-percutaneous nephrolithotomy (PCNL). IBJU 40: 568-573.

12. N Anil Kumar, S Venkata Chaitanya, Satish Bezawada, SR Sree Gouri (2016) Post percutaneous nephrolithotomy massive hematuria: Our experience. International Journal of Contemporary Medical Research 3: 1499-1502.

13. Guohua Zeng, Zhhenhua Zhao, Shawpong Wan, Sanjay Khadgi, Yongfu Long, et al. (2013) Failure of initial renal arterial embolization for severe post- percutaneous nephrolithotomy hemorrhage: A multicenter study of risk factors. J Urol 190: 2133-2138.

14. Qiqi Mao, Chaojun Wang, Geming Chan, Fuqing Tan, Bohua Shen (2019) Failure of initial superselective renal arterial embolization in the treatment of renal hemorrhage after percutaneous nephrolithotomy: A retrospective analysis of risk factors. Exp Ther Med 18: 4151-4156. 